\title{
Determinants of ejaculatory failure in the copulatory behavior of cactus mice (Peromyscus eremicus)
}

\author{
DONALD A. DEWSBURY \\ University of Florida, Gainesville, Florida 32611
}

\begin{abstract}
Although cactus mice, Peromyscus eremicus, typically resume copulatory behavior soon after attaining their first ejaculation, they rarely attain additional ejaculations. Experiment I was designed to determine the relative contribution of the male and female to this phenomenon of "ejaculatory failure" - the failure to attain ejaculation despite initiation of a series. Each of 18 male and 18 female cactus mice was tested in three conditions: once with a mated male paired with an unmated female, once with an unmated male paired with a mated female, and once with the original pairing retained. Ejaculatory failure was found to be primarily attributable to changes in the male. In Experiment II, it was found that the inhibitory effects of the first series are largely, but not completely, dissipated after $24 \mathrm{~h}$. The first ejaculatory series appears to produce an elevation of the male's ejaculatory threshold.
\end{abstract}

Rodent copulatory patterns can be described using a relatively small set of measures which are defined in terms of male behavioral patterns-mounts, intromissions, and ejaculations (e.g., Beach \& Jordan, 1956). In most species, these measures show orderly changes as an episode progresses. The fact that these measures, while defined in terms of male behavior, are a joint function of both male and female often is neglected (see Doty, 1974).

The relative role of the male and female in producing the orderly changes in behavior that unfold throughout an episode can be dissected by re-pairing mated and unmated males and females at selected times in the episode (Clemens, 1969; Dewsbury, 1968). Clemens showed that the failure of male deermice to show a reduction from the first to the second series in the number of intromissions required to attain ejaculation was indeed a function of the female rather than of the male.

An attempt to separate influences of the male and female on copulatory behavior would be of particular interest in cactus mice, Peromyscus eremicus, because they frequently display the phenomenon of "ejaculatory failure." As with many rodent species, cactus mice display three fundamental copulatory behaviors: mounts (wherein the male mounts the female but does not gain vaginal penetration), intromissions (wherein mounting leads to penetration but not ejaculation), and ejaculations (with both penetration and ejaculation). These events are organized into "ejaculatory series," with each series containing one or more intromissions, ending with an ejacula-

Supported by Grant BMS75-08658 from the National Science Foundation. tion, and followed by a postejaculatory refractory period. Intravaginal thrusting occurs during intromissions and ejaculations (Dewsbury, 1974a). In contrast to many rodent species, cactus mice rarely attain more than one ejaculation. The first ejaculation usually is followed by an incomplete series, which typically includes more copulatory activity than the complete series with ejaculation. For example, one group of mice attained ejaculation after a mean of 10.7 intravaginal thrusts in the first series, but failed to attain ejaculation in subsequent incomplete series that contained a mean of 28.5 thrusts (Dewsbury, 1974a). This failure to attain ejaculation despite the initiation of an ejaculatory series is here termed "ejaculatory failure." It is treated as a normal part of the copulatory pattern in this species and is not intended to imply any abnormality.

Although the phenomenon of ejaculatory failure appears relatively rare in laboratory rats (e.g., Beach \& Jordan, 1956), it is by no means unique to cactus mice, as it comprises the dominant mode of attaining sexual satiety in montane voles (Dewsbury, 1973), Mongolian gerbils (Davis, Estep, \& Dewsbury, 1974), and California mice (Dewsbury, 1974b). Further, the copulations which comprise incomplete series are not without biological significance; they are critical to the initiation of successful pregnancy in cactus mice (Dewsbury \& Estep, 1975).

\section{EXPERIMENT I}

If ejaculatory failure is attributable to changes in the male, introduction of an unmated female after the first ejaculation of an episode should produce no change in its occurrence, and unmated males 
Table 1

Postmanipulation Copulatory Behavior as a Function of Condition in Experiment I

\begin{tabular}{|c|c|c|c|}
\hline \multirow{2}{*}{ Measure } & \multicolumn{2}{|r|}{ Condition } & \multirow[b]{2}{*}{$\begin{array}{l}\text { Mated } \\
\wp(\mathrm{Mr})\end{array}$} \\
\hline & $\begin{array}{l}\text { Control } \\
\text { (C) }\end{array}$ & $\begin{array}{l}\text { Unmated } \\
q \text { (UF) }\end{array}$ & \\
\hline $\begin{array}{l}\text { Number with intromissions } \\
\text { without ejaculation }\end{array}$ & $14 / 18$ & $11 / 18$ & $3 / 18$ \\
\hline Number with IF $<2$ & $5 / 14$ & $2 / 11$ & $3 / 3$ \\
\hline Number with IF > 2 & $9 / 14$ & $9 / 11$ & $0 / 3$ \\
\hline Number with ejaculation & $1 / 18$ & $3 / 18$ & $10 / 18$ \\
\hline Number not copulating & $3 / 18$ & $4 / 18$ & $5 / 18$ \\
\hline $\begin{array}{l}\text { Mean postmanipulation } \\
\text { intromission latency* }\end{array}$ & 513.1 & 1547.8 & 1689.8 \\
\hline
\end{tabular}

*In seconds for all males attaining intromission

should not fail to ejaculate when tested with recently rnated females. If ejaculatory failure is a function of changes in the female, however, the introduction of an unmated female after one ejaculation should eliminate ejaculatory failure; and unmated males should demonstrate ejaculatory failure when paired with recently mated females. These possibilities were tested in Experiment I.

\section{Methods}

The subjects were 18 male and 18 female sexually mature cactus mice, Peromyscus eremicus, at least 90 days of age. These animals were descendents of the Rillito population described by Dewsbury (1974a) and were selected from an original pool of 46 male and 42 female cactus mice on the basis of their having copulated in pretests. Housing conditions were as described by Dewsbury (1974).

Females were brought into behavioral estrus with injections of $0.08 \mathrm{mg}$ estradiol benzoate 3 days before testing and $0.8 \mathrm{mg}$ progesterone $6 \mathrm{~h}$ before testing.

Tests were initiated about midway through the dark phase of the diurnal cycle by placing the female in the male's home rage. Mice were permitted $90 \mathrm{~min}$ to initiate copulation as intromission latencies in this species tend to be quite long (Dewsbury, 1974a). If copulation was initiated and no experimental intervention was scheduled, tests were continued until the pair attained satiety criterion of 30 min with no intromissions.

In addition to the positive pretest, each male received three tests, with a minımum of 2 weeks intervening between successive tests. In one test, the control condition (C), the female was removed from the male's cage within $1 \mathrm{~min}$ of the occurrence of ejaculation and was immediately reintroduced to the cage. This manipulation was to control for effects of handling per se. In the second condition, the unmated female condition (UF), the first female was replaced within I min of the ejaculation by a second injected female which had not been mated in at least 2 weeks. In the final condition, the mated female condition (MF), the male was paired at the start of nis test with a female that had just completed one ejaculatory series with a different male.

Each male was tested once in each condition, with test order varied for different anımals so that all six possible orders were represented.

The following measures were taken: Intromission latency (IL), latency from introduction of the female to the first intromission; ejaculation latency (EL), latency from the first intromission of a series to tts terminal ejaculation; intromission frequency (IF), the number of intromissions in series; intromission thrust frequency (IIF), the number of deep thrusts on all intromiscions of a series; intromission durat on per intromisuion (ID I), mean duration of the intromissions of a series, mean inter-intromission interval (MII). mean interval separating the intromissions of a series; and postejaculatory interval (PEI), the interval from ejaculation to the next intromission. Where a measure is placed in quotation marks, it refers to an incomplete series (i.e., one not ending in ejaculation, but excluding the 30-min criterion terminating the episode).

\section{Results}

The results of this experiment are summarized in Tables 1 and 2. The data for condition $C$ generally fall within the range of those reported by Dewsbury (1974a). The mean postmanipulation intromission latency, which, in this case, is essentially equivalent to a PEI, was approximately $81 / 2 \mathrm{~min}$. Only three males failed to initiate second series and just one male attained a second ejaculation. Fourteen of the 18 males displayed ejaculatory failure. Most, 9 of 14 , achieved more than two intromissions, with a mean 11.4 intromissions for all males displaying ejaculatory failure.

Postmanipulation behavior of males in conditions MF and UF can be compared with that in condition $\mathrm{C}$. Such comparisons center about the first series of an episode in condition MF and postejaculatory behavior in conditions UF and C. A majority of males tested in condition UF, like condition $\mathrm{C}$, showed ejaculatory failure. By contrast, just 3 of the 18 males displayed ejaculatory failure when tested in condition MF. The difference in incidence of ejaculatory failure among the three conditions was significant when tested with a Cochran $Q$ test $(Q=12.93$, df $=2, \mathrm{p}<.01)$ (Siegel, 1956). Individual comparisons with McNemar tests (Siegel, 1956) revealed that condition MF differed from both condition UF $\left(\chi^{2}=6.12, p<.02\right)$ and condition $C\left(\chi^{2}=7.69\right.$, $\mathrm{p}<.01)$ but that the latter two conditions did not differ from each other $\left(\chi^{2}=0.44\right)$.

A further indication of the differences among these groups can be seen in the number of intromissions achieved in the incomplete series that did

Table 2

Mean Scores in Measures of Postmanipulation Copulatory Behavior for Incomplete Series in the Control and Unmated Female Conditions of Experiment I

\begin{tabular}{lrrr}
\hline & \multicolumn{3}{c}{ Condition } \\
\cline { 2 - 4 } Measure & Control (C) & Unmated (UF) & \multicolumn{1}{c}{$\mathrm{t}$} \\
\hline Number of tests & 14 & 11 & \\
$\mathrm{IL} \leftarrow$ (postmanipulation) & 526.4 & 1624.6 & 2.19 \\
$\mathrm{EL} \dagger$ & 1078.9 & 1443.4 & .75 \\
$\mathrm{IF}+$ & 11.4 & 13.0 & .32 \\
$\mathrm{ITF}+$ & 20.6 & 22.5 & .05 \\
$\mathrm{ID} / \mathrm{I}^{\dagger}$ & 2.7 & 2.6 & .05 \\
MIII & 222.6 & 174.6 & .53 \\
\hline
\end{tabular}

$*_{p}<0.5 \quad$ tMcasured in seconds
.75 (1) , 
occur. While most of the males displaying ejaculatory failure in conditions $\mathrm{C}$ and UF attained more than two intromissions, none of the males displaying ejaculatory failure in condition MF attained more than two intromissions.

Data on ILs revealed quite a different trend. Analysis of variance showed a significant difference in IL as a function of condition $(F=4.79$, $\mathrm{df}=2,39, \mathrm{p}<.05$ ). ILs in condition $\mathrm{C}$ were shorter than those in either condition UF $(t=2.34$, $\mathrm{df}=27, \mathrm{p}<.05)$ or condition MF $(\mathrm{t}=4.55$, $\mathrm{df}=26, \mathrm{p}<.001)$. There was no difference between conditions UF and $M F(t=0.27, d f=25)$.

Quantitative data for the incomplete series for tests in which ejaculatory failure was displayed in conditions $\mathrm{C}$ and UF are presented in Table 2. Measures in these conditions were compared using $t$ tests. Because ejaculatory failure occurred in just three tests in condition MF, these data are not included and inferential statistics were not used for this condition. As with the data in Table 1, which included the males that ejaculated, there was a significant effect of condition on IL. However, the groups $\mathrm{C}$ and UF did not differ significantly on any other measures.

\section{EXPERIMENT II}

In Experiment I, it was determined that there is an effect of the first ejaculatory series on the male. It would be of interest to determine the time course of the dissipation of this effect. In rats, where the first series produces a general facilitation of copulatory behavior, the time course of the dissipation of the facilitatory effect has been determined in detail (Beach \& Whalen, 1959; Larsson, 1961). Experiment II was designed to provide some indication of the rate of dissipation of the inhibitory effects of the first ejaculatory series in male cactus mice.

\begin{abstract}
Methods
Each of the males used in Experiment I received an additional two tests. Except as noted, basic maintenance and testing conditions were identical to those of Experiment I. The first test was terminated at the occurrence of the male's first ejaculation. The second test was conducted with a different female, unmated for at least 2 weeks, and was initiated $24 \mathrm{~h}$ after the initiation of the first test. This permitted an anlysis of any inhibitory effects of the first ejaculatory series still present after $24 \mathrm{~h}$, which was unconfounded by changes correlated with diurnal cycles.
\end{abstract}

\section{Results}

Of the 18 males in this experiment, $11(61 \%)$ displayed at least one complete ejaculatory series in the second test, $5(28 \%)$ failed to copulate, and $2(11 \%)$ displayed ejaculatory failure as they achieved intromissions without ejaculation. The 2 males show- ing ejaculatory failure achieved 40 and 61 intromissions, respectively. Eight of the 11 males which ejaculated in the second test initiated second series, but none attained ejaculation. For those 8 males, there was a mean of 21 intromissions with 34 thrusts in the incomplete second series, thus illustrating the typical second-series ejaculatory failure syndrome.

\section{DISCUSSION}

These data indicate that, while both male and female may play a role in the changing interaction that occurs during an episode of copulatory behavior in cactus mice, ejaculatory failure is primarily a function of changes in the male. Ejaculatory failure was displayed by $61 \%$ of the males mating with previously unmated females. There was no significant effect on quantitative measures attributable to the female change. By contrast, the baseline rate for ejaculatory failure in the first series in rested pairs in the 68 tests (excluding pretests) that comprised these experiments was $4 \%$. In all of these cases, females were resistant and IF was three or less. Because males consistently engage in more copulatory activity than is sufficient to produce ejaculation in the first series, the conclusion that there is an elevation in the male's threshold for ejaculation as a consequence of the first series seems warranted.

Much of the inhibitory effect of one ejaculatory series was dissipated in $24 \mathrm{~h}$. Thus, $61 \%$ of the males were able to ejaculate after $24 \mathrm{~h}$. The eight males that initiated second series on the second day showed no reduction in number of intromissions and thrusts attained relative to the data of Experiment I and of Dewsbury (1974a). However, there is some evidence of a lack of complete recovery in at least some animals. The $28 \%$ noncopulating and $11 \%$ ejaculatory failure rates compare with base rates for this group of mice of $16 \%$ and $4 \%$, respectively. The "IFs" of 40 and 61 contrast sharply with those in the rare cases of ejaculatory failure in rested pairs (which never exceeded 3). It seems reasonable to conclude that while most males appear completely recovered from the effects of one ejaculatory series after $24 \mathrm{~h}$, inhibitory effects still are detectable in some animals.

Disturbance created by handling the female after ejaculation (condition C) appeared to have produced but slight interference with later copulatory behavior when compared to the data of Dewsbury (1974a), collected under similar testing conditions.

When unmated males were paired with recently mated females, the animals failed to copulate in $28 \%$ of the tests compared with a base rate for rested pairs of $16 \%$. Thus, there was a very slight elevation 
in failures to mate. Once copulation was initiated, however, ejaculation followed in $77 \%$ of the instances. Of those few males displaying ejaculatory failure, none showed the full syndrome which characterizes this species; none attained more than two intromissions. The latency to initiate copulation was greater than in the control condition, indicating that the male plays some role in controlling the characteristically long intromission latencies. The primary way in which this group appeared to differ from the other two was with respect to female resistance. As noted by Dewsbury (1974a), females become increasingly resistant after the first series. Female resistance appeared further increased as a function of re-pairing. Some females were so resistant that males which regularly copulated with unmated females were unable either to initiate copulatory activity or to attain more than a few intromissions. Female resistance clearly is an important influence on behavior after the first ejaculatory series. However, it is not a primary determinant of the phenomenon of ejaculatory failure as described here; that is due to a change in the male.

Replacement of the original mated female with an unmated female after the male's ejaculation produced a significant increase in the latency to the next intromission and was the only appreciable behavioral change created by the change of female. This may indicate that the female probably plays an important role in controlling the long intromission latencies which characterize this species (Dewsbury, 1974a). Alternatively, the male may require a period of time 10 interact with a novel conspecific before initiating copulatory activity.

The adaptive significance, if any, of this threshold elevation system is unclear. Females clearly need the stimuli derived from prolonged copulation in order to display neuroendocrine responses essential for pregnancy (Dewsbury \& Estep, 1975). They may receive enough sperm from one ejaculate for maximal fertilization. The threshold elevation may thus permit male cactus mice to deliver the vaginal stimulation required for successful pregnancy in females, without wasting sperm stores.

\section{REFERENCES}

BEACH, F. A., \& JoRdAN, L Sexual exhaustion and recovery in the male rat. Quarterly Joumal of Experimental Psychology. 1956, 8, 121-133.

Beach. F. A.. \& Whalen R. E. Effects of ejaculation on sexual behavior in the male rat. Journal of Comparative and Phvsiological Psichology. 1959. 52. 249-254.

Clemens, L. G. Experimental analysis of sexual behavior of the deermouse Peromyscus maniculatus gambeli. Behaviour, 1969 . 34, 267.285 .

Davis, H. N.. Estep, D. Q., \& Dewsbury, D A. Copulatory behavior of Mongolıan gerbils (Meriones unguiculatus]. Animal Learning \& Behavior, 1974, 2. 69-73.

Dewsbury, D. A. Copulatory behavior in rats: Changes as satiety is approached. Psychological Reports, 1968, 22. 937-943.

DEwsBURY, D. A. Copulatory behavior of montane voles (Microtus montanus). Behaviour. 1973, 44, 186-202.

Dewsbury, D. A. Copulatory behavior of wild-trapped and laboratory-reared cactus mice (Peromyscus eremicus) from two natural populations. Behavioral Biology, 1974, 11, 315-326. (a)

Dewseury, D. A. Copulatory behavior of Californa mice (Peromyscus calitornicus). Brain. Behavior, and Evolution, 1974, 9, 95-106. (b)

Dewsbury. D. A.. \& Estep. D. Q. Pregnancy in cactus mice: Effects of prolonged copulation. Sclence, 1975, 187. 552-553.

Doty. R. L. A crv tor the liberation of the female rodent: Courtship and copulation in Rodentia. Psychological Bulletin. 1974. 81. 159-172.

LARSSON. K. Duration of taclitatory effects of ejaculation on sexual behavior in the male rat. Journal of Comparatve and Physiological Psycholog. 1961, 54. 63-67.

Parker. G A Sperm competition and its evolutionary consequences in the insects. Biological Review's. 1970. 45. 525.567

SIEGEL. S Nonparametnc statistics for the behavioral sciences. Neu York: McGrau-Hill, 1956.

(Received for publication July 28, 1975; revision accepted October 22, 1975, 